\title{
PC-APC Schemes in Multipath Diversity System to Get Higher Throughput
}

\author{
Sanjit Ningthoujam, Manash Pratim Dutta, Subhasish Banerjee, Chandan T. Bhunia, \\ Swarnendu K. Chakraborty \\ Department of Electronics and Computer Engineering, National Institute of Technology, Arunachal Pradesh, India
}

\begin{tabular}{l}
\hline \hline Article Info \\
\hline Article history: \\
Received Aug 8, 2016 \\
Revised Jan 19, 2017 \\
Accepted Jan 31, 2017 \\
\hline Keyword: \\
APC \\
ARQ \\
BEC \\
ELA post reception selection \\
Combining \\
PC \\
Selection combining \\
\hline
\end{tabular}

\begin{abstract}
This paper is studied about a new protocol of Packet combining (PC) and Aggressive Packet Combining Scheme (APC) in multipath diversity system to get higher throughput. In the proposed protocol of PC and APC schemes, two and three copies of a packet are sent in two and three paths. If either of the copies is received without any erroneous then select the correct one and discard all other copies. Again if all copies are found as erroneous then combine the erroneous copies and perform XOR operation in case of PC and bit by bit majority logic in case of APC in order to get the original copy. The paper is implemented using Mat lab and found that new proposed protocols are getting higher throughput and probability of receiving successful packet at the receiver side. Thus, performance of error prone wireless network can be improved by applying either PC or APC in multipath diversity system.
\end{abstract}

Copyright (C) 2017 Institute of Advanced Engineering and Science. All rights reserved.

\section{Corresponding Author:}

Sanjit Ningthoujam,

Department of Electronics and Computer Engineering,

National Institute of Technology, Arunachal Pradesh, India 791112.

Email: sanjit.ningthoujam@gmail.com

\section{INTRODUCTION}

Automatic Repeat Request (ARQ) techniques are mainly implemented for error control by retransmitting duplicate copies in wireless network. Normally, the receiver discards all erroneous copies and request for retransmission. However, an erroneous packet may contain both erroneous and correct bits and hence the packet may contain useful information. Therefore, to get the correct information, it is deployed by combining multiple erroneous copies at the receiver side. S.Chakraborty et.al [1] suggested that PC is one of the simplest implementation of space diversity reception for combating fading in wireless communication system. In PC technique, only one error can be corrected and therefore, if double errors are found at same bit location of two copies then PC technique is not useful. Therefore, retransmission of duplicate packet has been occurred. Moreover, APC was introduced by Leung [2] for error correction and optimizes Backward Error Correction (BEC). Many researchers [3-14] have been studied and conclusively established that PC techniques provide higher throughput but low error correction capability to that of APC schemes. The error prone wireless network having bit error rate varies from $10^{-4}$ to $10^{-2}$ is neither reason of assuming nor any logic to apply only single path in case of conventional PC and APC techniques. Therefore, in the paper new protocol of PC and APC are introduced in order to reduce the dependency of a single path/route which gives more stable in wireless netwok.

The remaining of the paper is organized as follows. Reviews of conventional PC, APC and ELAPOR-SC are introduced in Section 2. Section 3 discusses about new protocols of PC and APC and Section 4 describes mathematical analysis of throughput and probability of error. Section 5 illustrates the simulation result using Mat lab and finally, conclusion is discussed in Section 6. 


\section{PRELIMINARIES}

\subsection{Packet Combining Scheme (PC)}

PCis proposed to locate erroneous bits received by the receiver.The technique of PC scheme are given here: let original packet be "00001111". Instead of these bits, the copy is received erroneously as "00001110". The receiver requests for duplicate copy after storing of received erroneous copy. Again, it is assumed that receiver received the copy as "00011111", which is also an erroneous. By combining erroneous copies at the receiver using XOR operation, it can identify the error location. The example is given here;

00001110

00011111

XOR 00010001

From the above example, $4^{\text {th }}$ and $8^{\text {th }}$ bit positionsfrom MSB are identified as erroneous bits. To get original packet by Chakraborty, the erroneous bits are converting received"1" to "0" or vice versa. Again, let original Packet as "11110000"

At the receiver

$1^{\text {st }}$ erroneous copy: 10110000 (erroneous occurs at $2^{\text {nd }}$ bit position from MSB)

$2^{\text {nd }}$ erroneous copy: 10110000 (erroneous occurs at $2^{\text {nd }}$ bit position from MSB)

XORed 00000000

The operation cannot be identified erroneous bit position when erroneous occurs at same bit position. So, it is failed to correct erroneous copy.

\subsection{Aggressive Packet Combining Scheme (APC)}

APC is a modified version of Majority Packet Combining. This technique isused for obtaining high error correction capability in wireless networks. Examples of APC are given here:

a. "00111" is assumed as original packet which is sent form sender to receiver. It is also assumed that the original packet is received as erroneous.Therefore, the receiver request for duplicate copies from sender. Let all the copies are received with error as: $1^{\text {st }}$ erroneous copy: 00011, $2^{\text {nd }}$ erroneous copy : 00110 and $3^{\text {rd }}$ erroneous copy:00011.

b. 00011 are generated at the receiver side after performing bit by bit majority logic on three erroneous copies.

c. Error detection scheme is applied at receiver to check the generated copy is original or not. If it is not actual packet, least reliable bits will be chosen from majority logic. Therefore, in the example the $3^{\text {rd }}$ and $5^{\text {th }}$ bit from the MSB is chosen.

Brute force correction method is applied as in PC technique to the $3^{\text {rd }}$ and $5^{\text {th }}$ bits, followed by error correction method. This process may lead to get actual packet. If actual packet is not generated in the above process then it will discard all erroneous copiesandrequests for duplicate copies.

\subsection{Error Location Algorithm-POR-SC}

Liang et.al [5] introduced Error Location Algorithm (ELA) which is an enhanced version of Automatic Repeat Request-POR-SC. The scheme operates as follow: if any one of the received copies is correct, then it is accepted. However, if all copies are erroneous then in order to get correct copy, it performed ELA-POR-SC. In this scheme, three copies are sent from transmitter to receiver. At least one copy is found as correct then proceed for the next packet otherwise performing ELA-POR-SC. It is assumed that $\mathrm{A}, \mathrm{B}, \mathrm{C}$ are the three received of erroneous copies of the same transmitted packet from transmitter over three diversity channels, then:

$\mathrm{AA}=(\mathrm{A}$ xored $\mathrm{C}) .(\mathrm{A}$ xored $\mathrm{B})$

$\mathrm{BB}=(\mathrm{A}$ xored $\mathrm{B}) \cdot(\mathrm{B}$ xored $\mathrm{C})$

$\mathrm{CC}=(\mathrm{A}$ xored $\mathrm{C}) .(\mathrm{B}$ xored $\mathrm{C})$

In $\mathrm{AA}, \mathrm{BB}$, and $\mathrm{CC}$, every ' 1 ' indicates an error in the copy of $\mathrm{A}, \mathrm{B}$ and $\mathrm{C}$ respectively. In this case, the original packet can be retrieved by using

$\mathrm{S}=\mathrm{AA}$ xored $\mathrm{A}(=\mathrm{BB}$ xored $\mathrm{B}=\mathrm{CC}$ xored $\mathrm{C})$

However, the retrieval process fails when it is found as double, triple error and so on. In this time, erroneous copies are discarded and request for next duplicate copies by sending NAK. 
Table 1. Examples of ELA-POR-SC.

\begin{tabular}{lcc}
\hline Original Packet at Sender & \multicolumn{2}{c}{ Receiver (Erroneous bits are underlined) } \\
\hline 1.11100011 & $\mathrm{~A}-11110011$ & $\mathrm{AA}=00010000$, \\
& $\mathrm{B}-\underline{0} 1100011$ & $\mathrm{~S}=11100011$ (Original \\
& $\mathrm{C}-1110001 \underline{0}$ & packet) \\
2.00001111 & $\mathrm{~A}-01001111$ & $\mathrm{BB}=10000000$, \\
& $\mathrm{B}-10001111$ & $\mathrm{~S}=00001111($ Original \\
& $\mathrm{C}-000011 \underline{0} 1$ & packet) \\
3.10101010 & $\mathrm{~A}-101010 \underline{0} 0$ & $\mathrm{CC}=00001000$, \\
& $\mathrm{B}-1 \underline{1} 101010$ & $\mathrm{~S}=10101010($ Original \\
& $\mathrm{C}-1010 \underline{0} 010$ & packet) \\
\hline
\end{tabular}

\section{PROPOSED PROTOCOLS}

\subsection{Proposed Protocols of PC Scheme}

In this new PC scheme, duplicate copies of a same packet are transferredfrom sender to receiver by using two paths (branches). The step wise procedures are illustrated below:

a. The first copy is sent original as " 11100011 " in first path but erroneously received as 10100011 .

b. As in PC scheme, duplicate copy will be sent in second path by shifting three bits from LSB to MSB as 01111100. It is assumed that the copy has been found error at $2^{\text {nd }}$ bit position from MSB as 01111100 . In order to get the original bit sequence, the received copy will be shifted back from MSB to LSB by three bits at the receiver side as 11100011 .

c. The two erroneous copies are stored and performed XOR operation to locate the erroneous bit position and apply brute force method as conventional PC scheme (erroneous bits are underlined). The example is given below:

$$
\begin{aligned}
& \text { Original packet as " } 11100011 \text { " } \\
& 1^{\text {st }} \text { erroneous copy- } 1 \underline{0} 100011 \\
& 2^{\text {nd }} \text { erroneous copy- } 111000 \underline{1} 1
\end{aligned}
$$$$
\text { XORed } 01000010
$$

This technique overcomes the limitation of conventional PC as receiver will be detected same bit error location in two erroneous copies.

\subsection{Proposed protocol of APC}

In this protocol, triplicate copies of same packet are sent in three paths from sender to receiver. The stepwise procedures are illustrated below:

a. The first copy is sent in first path as original packet.

b. The second copy will be sent after three bits shifting from LSB to MSB in second path. The receiver will perform three bits shifting from MSB to LSB.

c. The third copy will be sent in third path after six bits shifting from LSB to MSB and at receiver side the six bits will be shifted back from MSB to LSB in order to get the original sequence of bits position.

d. The three copies are stored in receiver and perform Aggressive Packet Combining Scheme to get the

\begin{tabular}{|c|c|c|c|}
\hline \multicolumn{2}{|c|}{ Original Packet } & Sender & Receiver (Erroneous bits are underlined) \\
\hline 1. & 11110011 & $\begin{array}{l}1^{\text {st }} \text { copy }-11110011 \\
2^{\text {nd }} \text { copy }-01111110 \\
3^{\text {rd }} \text { copy }-11001111\end{array}$ & $\begin{array}{l}1^{\text {st }} \text { copy }-\underline{-00110011} \\
2^{\text {nd }} \text { copy- } 11110 \underline{101} \\
3^{\text {rd }} \text { copy }-11000011\end{array}$ \\
\hline 2. & 11110000 & $\begin{array}{l}1^{\text {st }} \text { copy }-11110000 \\
2^{\text {nd }} \text { copy }-00011110 \\
3^{\text {rd }} \text { copy }-11000011\end{array}$ & $\begin{array}{l}11110011 \text { (original packet after majority packet combining) } \\
\qquad \begin{array}{l}1^{\text {st }} \text { copy- } \underline{0} 1110001 \\
2^{\text {nd }} \text { copy }-1111100 \\
3^{\text {rd }} \text { copy- } 10010000\end{array}\end{array}$ \\
\hline 3. & 00001111 & $\begin{array}{l}1^{\text {st }} \text { copy }-00001111 \\
2^{\text {nd }} \text { copy }-11100001 \\
3^{\text {rd }} \text { copy }-00111100\end{array}$ & $\begin{array}{l}11110000 \text { (original packet after majority packet combining) } \\
\qquad 1^{\text {st }} \text { copy }-00010111 \\
2^{\text {nd }} \operatorname{copy}-11001111 \\
3^{\text {rd }} \text { copy }-\underline{00001111}\end{array}$ \\
\hline & & & 00001111 (original packet after majority packet combining) \\
\hline
\end{tabular}
original packet.The examples of proposed protocols are illustrated as below:

Table 2. Erroneous may happen at any two bits position 


\section{ANALYSIS}

It is assumed that the sender sends bit 1 to receiver using single path and receiver receives bit 0 instead of 1 . Let ' $\mu$ ' be the probability of receiving bit error (received 0 instead of 1 and vice versa) and therefore, $1-\mu$ be the probability of correction bit at the receiver side. For ' $n$ ' packet size, the probability of error correction is given by Equation 1 .

$\mathrm{PC}=(1-\mu)^{\mathrm{n}}$

And Probability of error is given by Equation (2)

$\mathrm{PE}=\left[1-(1-\mu)^{\mathrm{n}}\right]$

It is assumed that in proposed PC and APC the sender sends two and three copies of $\mathrm{n}$ packet size in two and three paths then the probability of receiving at least one correct copy [5] is given by Equation 3 and 4 .

$$
\begin{aligned}
& X=1-\left[1-(1-\mu)^{n}\right]^{2} \\
& Y=1-\left[1-(1-\mu)^{n}\right]^{3}
\end{aligned}
$$

Throughput [19] of proposed PC and Proposed APC will be given by Equations (5) and (6)

$\mathrm{THpc}=(1-\pi \mathrm{pc}) /(2+\pi \mathrm{pc})$

THapc $=(1-\pi \mathrm{apc}) /(3+\pi \mathrm{apc})$

Where $\pi \mathrm{pc}=\left[\left(1-(1-\mu)^{\mathrm{n}}\right)+\left(1-(1-\mu)^{\mathrm{n}}\right)\right] / 2, \pi \mathrm{apc}=\left[\left(1-(1-\mu)^{\mathrm{n}}\right)+\left(1-(1-\mu)^{\mathrm{n}}\right)+\left(1-(1-\mu)^{\mathrm{n}}\right)\right] / 3$

\section{RESULTS}

4.1. Comparisons of proposed APC and PC Schemes in Term of Probability of Successful Packet Received at the Receiver Side

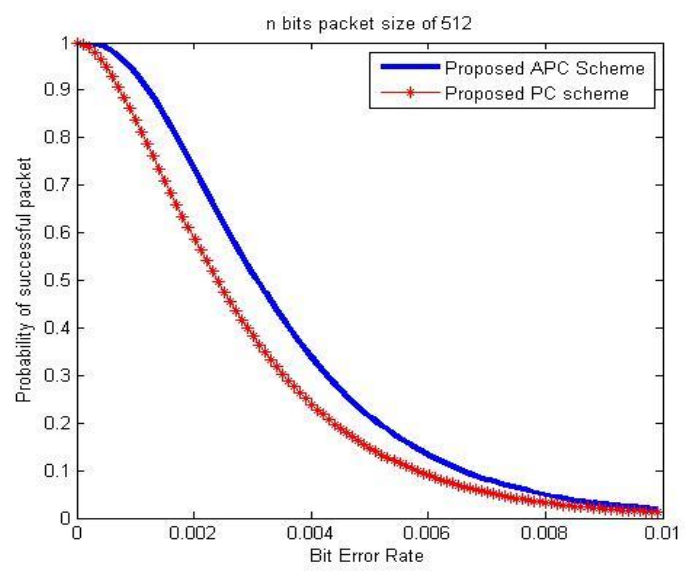

Figure 1. 512 Bits Packet Size

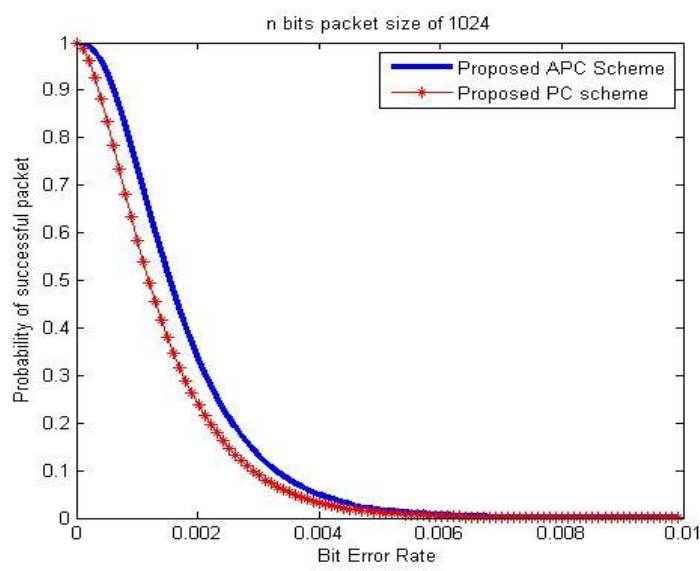

Figure 2. 1024 Bits Packet Size 


\subsection{Comparison of Proposed PC and Conventional PC Schemes in Term of Throughput}

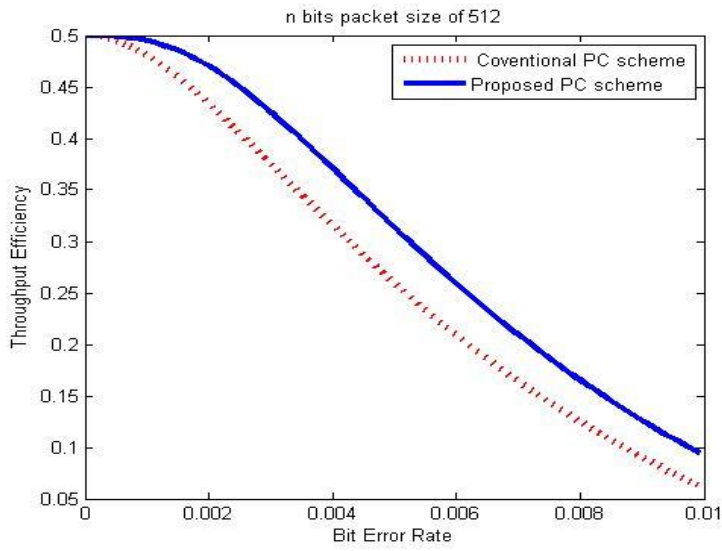

Figure 3.512 bits packet size

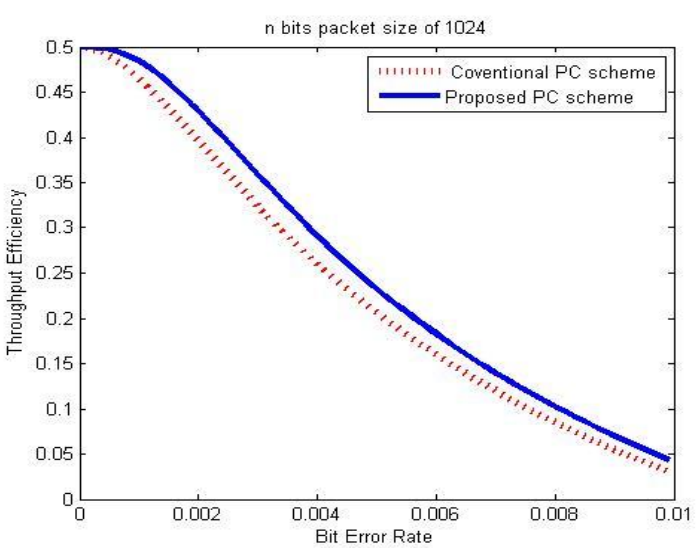

Figure 4. 1024 bits packet size

\subsection{Comparison of Proposed APC and Conventional APC Schemes in Term of Throughput}

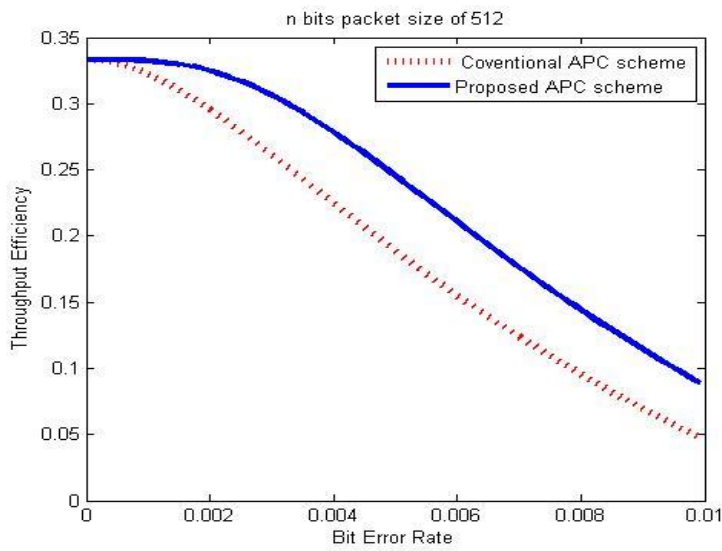

Figure 5.512 bits packet size

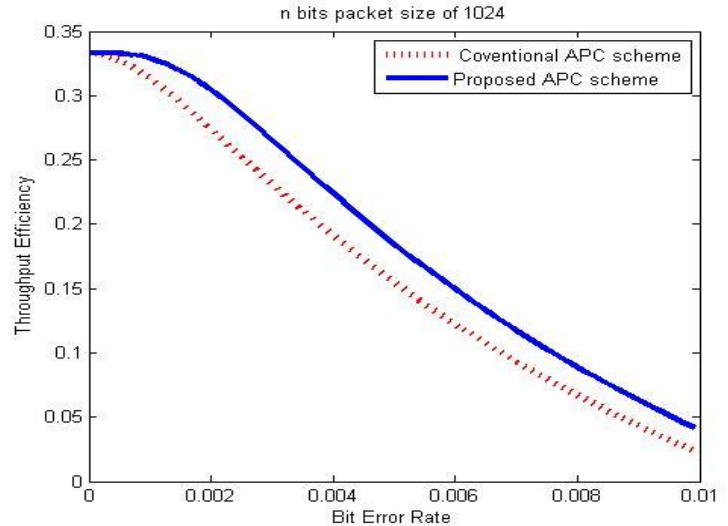

Figure 6. 1024 bits packet size

\section{CONCLUSION}

In wireless networks, data transmission is high chance to get failured and thus, retransmission of duplicate copies from sender to receiver may drastically reduce the throughput. In orderto reduce retransmission of duplicate copies, erroneous copies are combinedtoget original copy. The technique is done by PC and APC but they have low performance. Therefore,new proposed techniques are introduced to increase the performance of the conventional PC and APC. In the results, figure 1 and figure 2 clearly showed that comparison of proposed techniques of APC and PC in term of probability of receiving at least one correct packet at the receiver. In figures 3-6, it is also clearly evident that proposed PC and APC techniques using multipath provide higher throughput in bit error rate varies $10^{-4}$ to $10^{-2}$ and packet size from 512 to 1024 bits. Thus, multipath PC-APC is outperformed in terms of throughput and probability of receiving atleast one correct copy.

\section{REFERENCES}

[1] Shyam S. Chakraborty et al, An ARQ Scheme with Packet Combining, IEEE Comm Letters, Vol 2, No 7, July '95, pp 200-202. 
[2] Yiu-Wing LEUNG, Aggressive Packet Combining for Error Control in Wireless Networks, trans. Comm Vol. E83, No 2 Feb '2000, pp 38-385

[3] C Bhunia, A Few Modified ARQTechniques, Proceedings of the International Conference on Communications, Computers \& Devices, ICCCD-2000,14-16, Decedmber'2000, IIT, Kharagpur, India, Vol.II,pp. 705-708.

[4] CT Bhunia, ARQ Techniques: Review and Modifications, Journal IETE Technical Review, Sept-Oct '2001 Vol 18, No 5, pp 381-401.

[5] Liang, Y. and S. Chakraborty, S.S (2004) 'An ARQ and Packet Combining with Post-reception selection diversity', Proceedings of the IEEE Vehicular Technology Conference (VTC), Los Angeles, USA, PP. 1853-1857.

[6] Sanjit Ningthoujam, Swarnendu K Chakraborty, Chandan T Bhunia, "New Protocol for Aggressive Packet Combining in Gilbert Two State Model Using Back Up Routes to Achieve Higher Throughput", Proc. ICARCSET2015, ACM, ICPS, March, 6th - 7th 2015, Eluru, Andhra Pradesh, India.

[7] Sanjit Ningthoujam, Swarnendu K Chakraborty, Chandan T Bhunia, "New Investigation of Aggressive Packet Combining Scheme to Reduce Transmission Delay and Three States Markov Model using Multiple Routes to Increase throughput", Published to International Journal of Future Generation Communication and Networking, VOL 8, No.5 (2015), pp 157-166.

[8] Swarnendu K Chakraborty, Rajat S Goswami, Abhinandan Bhunia, CT Bhunia, "Investigation of two new protocols of Aggressive Packet Combining in achieving Better throughput", Published to Journal of the Institution of Engineers (India): Series B. 15 july 2014.

[9] Swarnendu K Chakraborty, Rajat S Goswami, Abhinandan Bhunia, CT Bhunia, "Studies of Several New Modifications of Aggressive Packet Combining to Achieve Higher Throughput, Based on Error Correction Capability of Disjoint Error Vectors ", Published to Journal of the Institution of Engineers (India): Series B. 30 January 2015.

[10] C T Bhunia,"Modified Aggressive Packet Combining Scheme", Pre-print, ICTP, Italy, IC/2010/037, pp. 1-10.

[11] Swarnendu K Chakraborty, Rajat S Goswami, Abhinandan Bhunia, Chandan T Bhunia, "New Protocol of Aggressive Packet Combining in Gilbert Two State Model", IEEE, CIEC, 2014, University of Calcutta.

[12] Swarnendu K Chakraborty, Rajat S Goswami, Abhinandan Bhunia, Chandan T Bhunia, "Two new modified schemes of Aggressive Packet Combining Schemes in Achieving Better Throughput", IEEE, ITNG, 2013,pp-729730 IEEE Computer Soc, Las Vegas.

[13] C T Bhunia, Modified Packet Combining Scheme using Error Forecasting Decoding to combat error in network, Proc. ICITA'05(Proc. IEEE Computer Soc.), Sydney, Vol, 2, 4-7, July '2005, pp 641-646

[14] CT Bhunia, Packet Reversed Packet Combining Scheme, Proc. IEEE Computer Soc, CIT'07, Aizu University, Japan, pp. 447-451.

[15] Don Towsley, The Shutter Go Back-N ARQ Protocol, IEEE Trans Comm, Vol 27, No 6, June'79, pp 869-875.

[16] RJ Beniece and AH Frey Jr, An analysis of retransmission schemes, IEEE Trans CommTech, COM-12, pp 135-145, Dec 1964

[17] S Lin, D Costello Jr and MJ Miller, Automatic repeat request error control schemes, IEEE Comm Mag, 22, pp 5-17, Dec '1984.

[18] ARK Sastry, Improving Automatic Repeat Request (ARQ) Performance on Satellite Channels Under High Error Rate Conditions, IEEE Trans Comm, April'77, pp 436-439.

[19] C T Bhunia, "Information Technology Network and Internet", New A.ge International Publishers, First Edition: 2005.

[20] Nabil Arman, Faisal Khamayseh "A Path-Compression Approach for Improving Shortest-Path Algorithms", International Journal of Electrical and Computer Engineering (IJECE, ISSN: 2088-8708), Vol 5, No 4, August 2015.

[21] A.B.M. Nasiruzzaman, "Complex Network Framework Based Comparative Study of Power Grid Centrality Measures”, International Journal of Electrical and Computer Engineering (IJECE, ISSN: 2088-8708), Vol 3, No. 4, August 2013.

\section{BIOGRAPHIES OF AUTHORS}

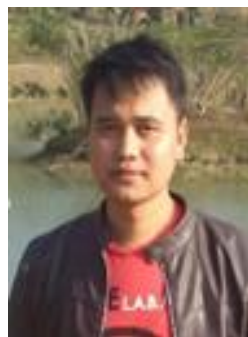

Sanjit Ningthoujam. He received B.Tech in Computer Science and Engineering from NERIST (Deemed University, Arunachal Pradesh) and also completed M.Tech in Mobile Communication and Computing under CSE Dept. at NIT Arunachal Pradesh. He is currently pursuing Ph.D in of Electronics and Computer Engineeringat NIT Arunachal Pradesh under the guidance of Dr. Swarnendu K Chakraborty. 

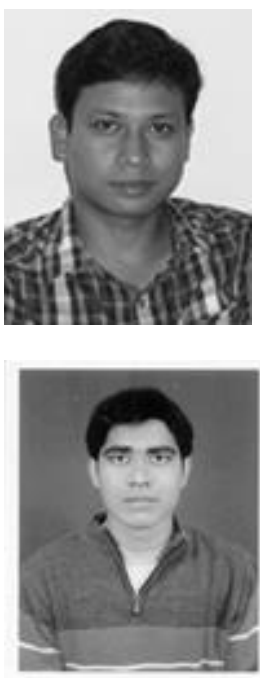

Manash Pratim Dutta. He received his M.Tech degree in Information Technology from Sikkim Manipal University, Sikkim, India in 2012. Currently, he is working as Assistent Professor and persuing his Ph.D in the Department of Computer Science and Engineering in National Institute of Technology, Arunachal Pradesh. His research activities are mainly focused on crytography and infornation secutity.

Subhasish Banerjee. He received his Ph.D in Computer Science and Engineering from National Institute of Technology, Arunachal Pradesh, in 2016 and M.Tech degree in Computer Application from Indian Institute of Technology(ISM), Dhanbad, India in 2012. Currently he is working as Assistent Professor in the Department of Computer Science and Engineering in National Institute of Technology, Arunachal Pradesh. His research activities are mainly focused on crytography and infornation secutity.

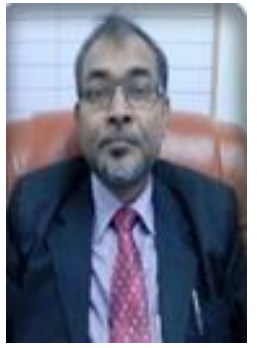

Chandan T Bhunia. Heearned his B.Tech in radio physics and electronics in 1983 from the Calcutta University, and then joined DVC of Govt. of India as telecommunication engineer. He got M.Tech in radio physics and electronics in 1985, and then joined North Bengal University as a lecturer of computer science \& application in 1988, and became Assistant Professor of electronics \& communication engineering at the North Eastern Regional Institute of Science \& Technology (NERIST) of Govt of India in 1990. He got his PhD in computer science \&engineering from the Jadavpur University. He became full Professor in 1997 at NERIST where he was HOD of ECE \& CSE for about 6yrs and Dean (Academics/Post Graduate Studies) for about 1.5 years. He then switched to private engineering colleges from 1999 to 2003 as HOD, Deputy Director and Director. Lastly, he was a full Professor of computer science \& engineering of the Indian School of Mines (Deemed University) of Govt of India. He was a senior Professor and Dy Director (Acad) of Haldia Institute of Technology. He has extensively visited foreign countries, namely China, Italy, Singapore, UK and Bangladesh on several assignments including BOYSCAST Fellowship and ICTP senior associate ship. He has published around 200 research papers and technical articles/reports in national/international journals/magazines/seminars. He is the author of the books a) "Introduction to Knowledge Management"published by Everest Publishing House, Pune in 2003 and b) "Information Technology, Network and Internet", published by the New Age International Publishers, New Delhi. He is Fellow of the IETE and the IE(I), and a senior member of the IEEE \& CSI.

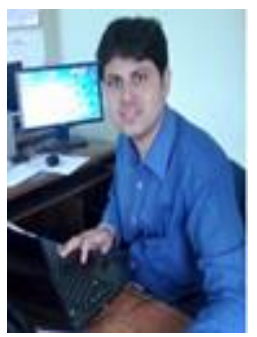

Swarnendu Kumar Chakraborty. He is working as an Assistant Professor \& HoD in the department of ofElectronics and Computer Engineeringat National Institute of Technology Arunachal Pradesh, Govt. of India. He is having more than 6 years of teaching experience. His research areas are advanced error control, cryptography and information security. He is the author of 22 peer-reviewed publications. In 2013, he has visited USA to present his one of article in ITNG 2013, he was awarded as best teacher for the academic year 2010-11. He received his $\mathrm{Ph} . \mathrm{D}$ from National Institute of Technology Arunachal Pradesh in 2015. 